\title{
Linx
}

Revue des linguistes de l'université Paris X Nanterre

39 | 1998

Modèles linguistiques : convergences, divergences

\section{Pierre Cadiot, Les prépositions abstraites du français}

Armand Colin, 1997

\section{Annie Montaut}

\section{CpenEdition}

\section{Journals}

Édition électronique

URL : http://journals.openedition.org/linx/931

DOI : 10.4000/linx.931

ISSN : 2118-9692

Éditeur

Presses universitaires de Paris Nanterre

\section{Édition imprimée}

Date de publication : 15 décembre 1998

Pagination : 260-264

ISSN : 0246-8743

\section{Référence électronique}

Annie Montaut, « Pierre Cadiot, Les prépositions abstraites du français », Linx [En ligne], 39 | 1998, mis en ligne le 02 juillet 2012, consulté le 22 septembre 2020. URL : http://journals.openedition.org/linx/931 : DOI : https://doi.org/10.4000/linx.931

Ce document a été généré automatiquement le 22 septembre 2020

Département de Sciences du langage, Université Paris Ouest 


\title{
Pierre Cadiot, Les prépositions abstraites du français
}

\author{
Armand Colin, 1997
}

Annie Montaut

\section{RÉFÉRENCE}

Pierre Cadiot Les prépositions abstraites du français, Armand Colin, 1997, 295p.

1 L'ouvrage propose une sémantique des prépositions abstraites ou «incolores» (de et à étant les plus longuement analysées) qu'il veut ouverte sur des questions générales reprenant, d'une façon très intéressante, car évitant toujours l'universalisme du cognitivisme, l'intérêt ancien pour les corrélats épistémiques et ontologiques de la sémantique prépositionnelle. L'auteur s'oppose à l'idée d'un sens propre qui s'attacherait aux prépositions en tant que mot (dont les emplois représenteraient les hyponymes de la valeur abstraite, dans une perspective verticale, ou bien les dérivés d'un sens prototypique, dans une perspective horizontale), sens qui généralement oppose le spatial au notionnel, le concret à l'abstrait, etc. L'auteur, s'attachant à voir plutôt cette polysémie structurée comme inscrite dans la syntaxe, montre tout au long des treize chapitres de l'ouvrage que les prépositions sont des instructions permettant de construire le référentiel, des modes donc de donation du monde référentiel, et non de simples renvois à ce référentiel, s'inspirant constamment du principe qu'il pose dès l'introduction: il s'agit «d'orientation, d'accès, de repérage, donc du sujet dans son rapport au monde, et non d'espace brut» (p.11). Claude Vandeloise avait déjà signalé l'importance de ce principe (ainsi «le livre est sur la table» ne correspond pas à «la table est sous le livre»), mais Cadiot l'articule à la recherche du type d'instruction que propose chaque préposition étudiée dans un contexte morpho-syntaxique donné. Vu le caractère crucial de ce contexte dans toutes les démonstrations, qui ancre le livre dans la langue et non dans l'épistémologie, j'en citerai des exemples concrets, quitte à allonger le compte-rendu. 
2 Après un chapitre qui fait le point sur la littérature ancienne et récente consacrée à la question, le chapitre 2, peut-être le plus lumineux sur le plan méthodologique parce qu'il pose les bases d'une opposition qui sera exploitée dans d'autres chapitres, s'oppose à l'illusion référentialiste que les prépositions puissent coder «les catégories de l'intuition localement explicatives», et propose comme "clé de l'alternance des prépositions à et de en contexte bi-nominal» (N1 à/de N2) l'opposition intension vs extension. Commençant par illustrer les vertus et les carences du principe physicaliste qui vouerait de à produire une image quantifiée du référent du syntagme N1 de N2, et à, à une image qualifiée de $\mathrm{N} 1$, il montre que à est une instruction intensionnelle, servant à fixer des propriétés de N1 («verre à vin»), alors que de est une instruction extensionnelle, l'accès au contenu par le contenant, voire la métonymie contenant/ contenu («avale ton verre de/*à vin») n'étant qu'un effet local du traitement extensionnel du SN. De en effet n'est pas tant associé à la quantification («pousse ton verre de vin») qu'à la saisie en extension, combinant les deux sites le GN et l'ensemble de l'énoncé, alors que à thématise N1. Ainsi le fait de pouvoir référer au contenant avec une certaine saisie du contenu, en contexte approprié, par une expression N1 à N2 («pousse ton verre à vin») invalide le principe physicaliste mais non sa reformulation en termes d'extension/intension. Même dans les cas où on pourrait se dire que à est plus affaire d'extension que d'intension («bouchon à dix $\mathrm{km}$ »), l'auteur montre que sa valeur, en fait prospective, à l'intention d'automobilistes en mouvement, plus que spatiale, relève de l'intension, et s'oppose justement à l'instruction extensionnelle de de («bouchon de dix km»).

3 Une des sections les plus intéressantes du chapitre porte sur la critique de la notion d'aliénabilité comme version matérielle de la relation partie/tout. Des contrastes comme «Paul a réparé /*regardé la voiture à Jean» [«Paul lui a réparé /*regardé la voiture» est encore plus clair, ne permettant pas la lecture 'voiture à Jean' comme équivalent de 'voiture de Jean»] vs «Paul a regardé sa voiture / la voiture de Jean», ou «Paul lui a repeint /*revu la maison», montrent que le datif n'est pas lié à la relation matérielle d'inaliénabilité comme on l'a souvent affirmé, et comme le donne à penser le contraste «Paul lui marche sur les pieds /*les chaussures»), mais au caractère intensionnel et programmé du prédicat. S'il se trouve que le possessif («de X, son») est caractéristique de la possession aliénable en pareil cas, c'est qu'il construit une relation en continu, et saisit ainsi la possession en extension, alors que le datif construit un «point de vue impliquant la partie dans sa relation non médiatisée avec le tout». A de s'attache un traitement extensionnel (continu), et à à une «mise en discontinu de la configuration référentielle et [...] une sémiotisation de ce discontinu avec effet de focalisation sur le sujet», en l'occurrence plutôt l'expérient, ou le possesseur, «et plus généralement de mise en perspective intensionnelle» (p. 57), <marcher sur les pieds> fonctionnant dans ce cas comme prédicat intensionnel restructuré vs. <marcher> dans les structures avec de. D'où les contrastes «son cœur bat», qui évoque, par la saisie extensionnelle, l'auscultation au cabinet médical, et «le cœur lui bat», qui dans sa saisie intensionnelle correspond à une description d'état psychologique, alors que «*les yeux lui pleurent» est peu acceptable, car il s'agit non de la description d'un affect, mais d'une affectation uniquement externe qui n'implique que l'organe et non le sujet, et donc s'accommode seulement de la saisie extensionnelle («ses»). Des contrastes comme «un ami à /de» sont aussi analysés dans cette perspective, reprise dans le chapitre 3, "dix thèses sur l'alternance à vs de en contexte verbal», notamment à propos de l'opposition aspectuelle : l'auteur y revient sur la valeur prospective de à comme saisie 
dynamique («revenir à de bons sentiments», «je t'obligerai à le faire»), opposé à la valeur factive, voire perfective, de de dans sa saisie statique («revenir d'une maladie», «tu n'étais pas obligé de le faire»).

4 Le long chapitre 4 sur les composés nominaux (N1 à N2), approche lexico-cognitive, procède par niveaux d'analyse (approche morphologique, syntaxique, la plus développée, sémiotique), tout en signalant qu'il ne saurait être question de les dissocier autrement que pour la clarté de la présentation. Là encore l'opposition extension/ intension est pertinente, par exemple dans l'occurrence du déterminant défini : «arbre aux/*à feuilles jaunies» relève de l'occurrence extensionnelle, alors que «arbre à /*aux feuilles caduques» construit une classe intensionnelle, établissant une sous classe sur la base d'une propriété significative dans une saisie référentielle unique du composé (d'où les effets d'«épure référentielle», N1 perdant une partie de son pouvoir descriptif). Une section particulièrement intéressante de ce chapitre concerne la distinction des deux types $\grave{a}=$ avec et $\grave{a}=$ pour («verre à pied» et «verre à dents»), outre le type métaphorique («pied à terre, sac à vin») et le type circonstanciel impliquant un scenario interprétatif («péage à pied»). l'auteur montre que l'interprétation du premier type saisit l'objet en le traitant comme une extension (et est donc privilégié pour les composés qui construisent une sous classe), il renvoie à une configuration réelle, alors que le second type (à=pour) renvoie à une configuration intensionnelle, saisissant l'objet à partir de son extérieur, notamment de ses fonctions. Les noms composés sont donc liés au traitement des connaissances encyclopédiques. Leur structure ne se borne pas à refléter des propriétés extralinguistiques mais enregistre «l'inscription dans la langue de propriétés suffisamment fermes et systématiques pour qu'on puisse les considérer comme sémantiques». Le nom composé, conclut l'auteur, promeut sémantiquement des propriétés qu'il homologue dans et par la langue (p.128), manière de voir tout-à-fait incontestable, interdisant de confondre linguistique et cognitivisme.

5 Le chapitre 5, «avec ou le déploiement de l'éventail», vise à cerner l'instruction abstraite donnée par la préposition, de manière à rendre compte de façon homogène d'emplois apparemment disparates voire contradictoires (comme la valeur de comitatif et d'opposant, «habiter avec» et «se battre avec»). l'auteur traite avec comme une instruction à créer «les conditions d'interaction optimales entre deux segments de réalité référés». Le chapitre contient quelques pages remarquables sur les protases, causales ou d'enchaînement illocutoire à valeur causale («il fallait qu'on le surveille, avec son accident», «avec le temps qu'il fait à Kathmandou, autant rester chez soi») ou concessive («on n'a pas gagné l'oscar, avec tous les efforts qu'on a faits»).

6 Comme il est impossible de rendre compte des autres chapitres (d'ailleurs plus brefs que les premiers) sans déborder les limites du compte-rendu, je me bornerai à en donner la liste : ils portent sur le contraste entre de et avec («toucher le tableau du doigt / avec la craie»), entre pour et une préposition «incolore» comme à/de («avoir le goût du théâtre, avoir du goût pour le théâtre»), les constructions «typicoprédicatives» («Karu est grand pour un japonais», «Médor est grand pour un pékinois»), les référents «improbables» de chez et en («il y a chez Proust une grande exigence», «il y a en Proust une grande rigueur»), les notions de prototype et de stéréotype («à propos de boîte»), «train et ses prépositions», la grammaire du mot vin, les prépositions dans les préparations et conditionnements culinaires.

7 Il faut pourtant, malgré tout le bien que j'ai pensé de ce livre et le plaisir qu'on prendra à sa lecture, signaler la négligence surprenante des finitions quant à la numérotation 
des exemples et aux renvois dans le texte à cette numérotation : alors que le livre ne présente pratiquement pas de fautes typographique (hormis les «raillures» de la $\mathrm{p}$. 132), des dizaines de renvois erronés gênent la lecture du texte. Ainsi dans le texte de la page 46 il faut lire (29) au lieu de (19) et (30) au lieu de (17), p. 56 il y a jusqu'à quatre erreurs de ce type, et l'ensemble de l'ouvrage est ainsi déparé, sans compter les erreurs d'astérisque («chemise à/*aux manches rapiécées» p. 104), de prénoms («ce sont bien des idées à Paul» glosé par «des idées typiques de Jean»p. 61, «Paul est un frère pour Paul» signalé comme déclenchant l'interprétation «Paul n'est pas un frère de Paul», ce qui, vu l'attribution des prénoms dans nos pays, relève d'autre chose que de la préposition et de sa syntaxe), etc.

Malgré ces erreurs regrettables dues probablement à des couper-coller intempestifs, l'ouvrage est remarquablement intéressant, se tenant d'un bout à l'autre à son principe de ne considérer les corrélats cognitifs de la sémantique prépositionnelle qu'à travers leur inscription dans la syntaxe. Il ne tombe ainsi jamais dans les dérives cognitivistes vers une logique abstraite du matériel linguistique fréquemment regrettables dans ce genre d'entreprise, tout en réussissant à articuler dans la logique cognitive les solutions qu'il propose. C'est ainsi aussi bien les sciences cognitives que la description empirique linguistique qui se trouvent bénéficier de cette approche. 